\title{
Administração peridural de associações de morfina, xilazina e neostigmina em cadelas: resultados preliminares
}

\author{
Effects of epidural administration of morphine, xylazine and \\ neostigmine combinations in dogs: preliminary results
}

Maria Guadalupe Dias Pestana Santos ${ }^{[a]}$, Eduardo Raposo Monteiro ${ }^{[b]}$, Gustavo Cancian Baiotto ${ }^{[c]}$, Renata Conti Ramos Teixeira ${ }^{[a]}$, Thaís Feres Bressan ${ }^{[a]}$, Daniela Campagno[ ${ }^{[b]}$

[a] Médicas-Veterinárias, mestrandas em Ciência Animal do Centro Universitário Vila Velha (UVV), Vila Velha, ES - Brasil, e-mails: lupitaa123@gmail.com; renatactp@yahoo.com.br; thais.bressan@hotmail.com

[b] Médicos-Veterinários, professores doutores do Mestrado em Ciência Animal do Centro Universitário Vila Velha (UVV), Vila Velha, ES - Brasil, e-mails: eduardo.raposo@uvv.br; daniela.campagnol@uvv.br

[c] Médico-Veterinário, professor mestre do curso de Medicina Veterinária do Centro Universitário Vila Velha (UVV), Vila Velha, ES - Brasil, e-mail: gbaiotto@uvv.br

\section{Resumo}

O objetivo do estudo foi verificar os efeitos cardiorrespiratórios e analgésicos da administração peridural de associações de morfina, xilazina e neostigmina em cadelas anestesiadas com meloxicam, acepromazina, morfina, propofol e isoflurano submetidas à mastectomia unilateral. Foram utilizadas 14 cadelas, divididas aleatoriamente em quatro grupos de acordo com os fármacos administrados via peridural: $0,30 \mathrm{~mL} / \mathrm{kg}$ de solução fisiológica ( $\mathrm{NaCl}$ 0,9\% - Grupo Controle: $\mathrm{n}=3$ ); 0,1 mg/kg de morfina (Grupo M: $\mathrm{n}=4$ ); 0,1 mg/kg de morfina $+0,01 \mathrm{mg} / \mathrm{kg}$ de neostigmina (Grupo MN: $\mathrm{n}=4$ ); 0,1 mg/kg de morfina + 0,1 mg/kg de xilazina (Grupo MX: $n=3$ ). 0 consumo de isoflurano durante a manutenção da anestesia, bem como as variáveis cardiorrespiratórias foram mensurados no período transoperatório e a dor foi avaliada no período pós-operatório por 24 horas. Nenhum dos tratamentos foi capaz de abolir a resposta hemodinâmica ao estímulo cirúrgico, caracterizada pela elevação na pressão arterial. No entanto, a administração dos tratamentos nos grupos M, MN e MX demonstrou tendência em atenuar essa resposta. A administração preemptiva de meloxicam e morfina sinalizou uma analgesia adequada em cadelas submetidas à mastectomia radical unilateral, porque não houve a necessidade de resgate analgésico nos grupos durante as primeiras 24 horas do período pós-operatório.

Palavras-chave: Analgesia preemptiva. Dor pós-operatória. Analgesia pós-operatória.

\section{Abstract}

The objective of the present study was to evaluate the cardiorespiratory and analgesic effects of the epidural administration of morphine, xylazine and neostigmine combinations. These combinations were studied in 14 female dogs anesthetized with meloxicam, acepromazine, morphine, propofol and isoflurane as they were submitted to unilateral mastectomy. The females were randomly divided into four groups according to the drugs 
administered epidurally: $0.30 \mathrm{~mL} / \mathrm{kg}$ of saline ( $\mathrm{NaCl} 0.9 \%$ - Control group: $n=3$ ), $0.1 \mathrm{mg} / \mathrm{kg}$ of morphine (Group M: $n=4), 0.1 \mathrm{mg} / \mathrm{kg}$ of morphine $+0.01 \mathrm{mg} / \mathrm{kg}$ of neostigmine (Group MN: $\mathrm{n}=4$ ), $0.1 \mathrm{mg} / \mathrm{kg}$ of morphine $+0.1 \mathrm{mg} / \mathrm{kg}$ of xylazine (Group MX: $n=3$ ). The requirement of isoflurane for maintenance of anesthesia and cardiorespiratory variables were measured during the intraoperative period and pain scores were evaluated during the postoperative period for 24 hours. None of the treatments was able to abolish the hemodynamic response to surgical stimulus, characterized by the increase of blood pressure. However, administration of treatments M, MN and MX appeared to attenuate this response. The pre-emptive administration of meloxicam and morphine appeared to provide adequate analgesia in dogs undergoing unilateral radical mastectomy, since there was no need for rescue analgesia during the first 24 hours after the operation.

Keywords: Preemptive analgesia. Postoperative pain. Postoperative analgesia.

\section{Introdução}

As neoplasias mamárias são comuns em cadelas, sendo que cerca de $35 \%$ a $50 \%$ delas são malignas. A remoção cirúrgica de tumores mamários é um dos procedimentos mais frequentes na rotina cirúrgica de cadelas adultas. A técnica de mastectomia pode ser parcial, simples, regional e radical unilateral ou bilateral (HEDLUND, 2005). A dor pós-operatória em cadelas submetidas à mastectomia radical pode ser caracterizada de moderada à intensa, sendo recomendado o tratamento com o uso de fármacos anti-inflamatórios não esteroidais associados aos opioides (CARROLL, 2005).

A administração de analgésicos pela via espinhal é uma das formas mais eficazes de prevenção e tratamento da dor. Quando comparada às vias intravenosa e intramuscular, a via espinhal geralmente utiliza doses menores dos fármacos, resultando em menor incidência de efeitos adversos (CARVALHO; LUNA, 2007). Dentre os fármacos utilizados pela via peridural, destaca-se a morfina, cuja administração resulta em analgesia de até 24 horas em média no período pós-operatório em cães (VALADÃo et al., 2002).

Além dos analgésicos opioides, outros fármacos vêm sendo utilizados pela via peridural, seja isoladamente ou em associação, tais como os agonistas alfa- 2 adrenérgicos e a neostigmina. Foi constatado que a xilazina, um fármaco da classe dos agonistas alfa-2 adrenérgicos, em associação ao anestésico local lidocaína, promove um bloqueio sensitivo mais cranial do que o uso isolado da lidocaína, possibilitando analgesia para cirurgias abdominais mais craniais (GASPARINI et al., 2007).
Os fármacos anticolinesterásicos, como a neostigmina, atuam inibindo a enzima acetilcolinesterase, responsável pela hidrólise da acetilcolina, aumentando o tempo de permanência desse neurotransmissor na fenda sináptica (SPINOSA, 2002). Seu emprego pela via peridural vem sendo evidenciado em seres humanos em associação a outros fármacos, como anestésicos locais e opioides, pois auxilia no controle da dor pós-operatória (LAURETTI et al., 1999). A provável ação dos anticolinesterásicos pela via espinhal se deve à ativação de receptores muscarínicos localizados na medula, havendo acúmulo de acetilcolina e da síntese de óxido nítrico (LOTHE et al., 1994). Em um estudo anterior realizado em cadelas submetidas à ovariosalpingohisterectomia, a administração peridural da neostigmina reduziu a necessidade de suplementação analgésica no período pós-operatório (MARUCIO et al., 2008). Entretanto, no mesmo estudo, o efeito analgésico da administração peridural da neostigmina foi menos pronunciado do que aquele proporcionado pela administração da morfina; e a associação da neostigmina não potencializou a analgesia mediada pela morfina. A hipótese para tal é que a dor após o procedimento cirúrgico é classificada de leve à moderada, não sendo possível detectar-se diferenças significativas entre os três tratamentos utilizados.

0 presente estudo teve por objetivo avaliar os efeitos cardiorrespiratório e analgésico da administração peridural das associações de morfina, xilazina e neostigmina nos períodos intraoperatório e pós-operatório de cadelas anestesiadas com isoflurano e submetidas à ovariosalpingohisterectomia e mastectomia radical unilateral. A hipótese do 
presente estudo foi que a neostigmina e a xilazina intensifiquem a analgesia proporcionada pela administração peridural da morfina.

\section{Materiais e métodos}

Este trabalho foi aprovado pela Comissão de Ética, Bioética e Bem Estar Animal do Centro Universitário Vila Velha CEUA-UVV (protocolo n. 51). Foram analisadas 14 cadelas, com idade média de 9,1 anos (variação de 6 a 13 anos), com peso médio de 9,1 kg, de raças variadas, submetidas à mastectomia radical unilateral e ovariosalpingohisterectomia, após permissão dos proprietários. No estudo, somente animais considerados hígidos (ASA I ou II) foram incluídos, com base em anamnese, exame físico, exames laboratoriais (hemograma completo com contagem de plaquetas, função hepática e renal), avaliação cardíaca e pulmonar por meio de eletrocardiograma e radiografia torácica. Os animais com alterações pré-operatórias ou com indícios de metástase pulmonar foram excluídos do estudo.

As cadelas foram submetidas à restrição alimentar pré-operatória de 12 horas, com acesso livre à água. A tranquilização foi realizada com acepromazina, na dose de $0,03 \mathrm{mg} / \mathrm{kg}$ e morfina, na dose de $0,5 \mathrm{mg} / \mathrm{kg}$ (IM). Um cateter 20 ou $22 \mathrm{G}$ foi inserido na veia cefálica para administração de fluídos e fármacos. Após 30 minutos da administração da tranquilização, a indução anestésica foi realizada com propofol (IV), na dose média de $4 \mathrm{mg} / \mathrm{kg}$. Subsequentemente, a traqueia foi entubada com uma sonda endotraqueal, a qual foi conectada a um circuito circular valvular, e a manutenção anestésica foi realizada com isoflurano.

Os animais foram ventilados mecanicamente, ajustando-se a frequência respiratória (FR) e a pressão inspiratória (Pinsp), de modo que a concentração expirada de dióxido de carbono $\left(\mathrm{ETCO}_{2}\right)$ fosse mantida entre 30 e $40 \mathrm{mmHg}$, sendo mensurada com o uso de um oxicapnógrafo conectado à extremidade distal da sonda endotraqueal. Durante todo o procedimento foi infundida solução de Ringer com lactato de sódio, na velocidade de $10 \mathrm{ml} / \mathrm{kg} /$ hora, por meio de uma bomba de infusão peristáltica. Todos os animais receberam administração IV de cefazolina (30 mg/kg) e meloxicam $(0,1 \mathrm{mg} / \mathrm{kg})$ previamente ao início do procedimento cirúrgico. A hipotermia foi atenuada preventivamente com o uso do colchão térmico.
Após um período de estabilização de dez minutos em anestesia com isoflurano, os animais foram inicialmente posicionados em decúbito ventral para a administração espinhal, utilizando uma agulha de Tuhoy, de um dos quatro tratamentos a seguir: Grupo Controle (Grupo C, $\mathrm{n}=3$ ), recebeu 0,3 ml/kg de solução salina ( $\mathrm{NaCl}$ 0,9\%); Grupo M (n=4) 0,1 $\mathrm{mg} / \mathrm{kg}$ de morfina; Grupo MX (n = 3) 0,1 mg/kg de morfina $+0,1 \mathrm{mg} / \mathrm{kg}$ de xilazina; Grupo $\mathrm{MN}(\mathrm{n}=4)$ $0,1 \mathrm{mg} / \mathrm{kg}$ de morfina $+0,01 \mathrm{mg} / \mathrm{kg}$ de neostigmina. A ordem dos tratamentos foi aleatória e em todos eles a padronização do volume foi feita a $0,3 \mathrm{ml} / \mathrm{kg}$ com adição de solução salina ( $\mathrm{NaCl}$ 0,9\%). Após a realização da anestesia peridural, os animais foram posicionados em decúbito dorsal.

Durante todo o procedimento cirúrgico, a concentração de isoflurano foi mantida inicialmente em 1,25\%, por meio de um vaporizador calibrado. A concentração vaporizada foi modificada de maneira a manter o animal em plano moderado de anestesia (globo ocular rotacionado, reflexo palpebral ausente, mandíbula relaxada). Um cateter 20 ou $22 \mathrm{G}$ foi introduzido de forma percutânea na artéria dorsal podal e conectado a um transdutor de pressão, cujo zero foi ajustado à altura do manúbrio do animal, para permitir a visualização de pressão arterial sistólica (PAS), média (PAM) e diastólica (PAD) na tela de um monitor multiparamétrico. As variáveis cardiorrespiratórias e a concentração vaporizada de isoflurano foram registradas antes do início da cirurgia (momento basal, aproximadamente 30 minutos após a administração peridural), e em intervalos de 15 minutos durante todo o procedimento cirúrgico (momentos M1 a M6), o qual foi realizado pelo mesmo cirurgião, seguindo rigorosamente a mesma técnica cirúrgica durante todo o estudo. Ao término do procedimento, a vaporização de isoflurano foi interrompida para permitir a recuperação anestésica dos animais. Os tempos de anestesia e cirurgia foram registrados.

A dor pós-operatória foi avaliada por um mesmo observador, sem ter sido informado sobre o tratamento peridural administrado. Para a avaliação da dor pós-operatória, utilizou-se a Escala Composta de Dor da Universidade de Glasgow (ECDUG, com variação de 0 a 10 - Tabela 1), modificada por Murrell et al. (2008). 0 grau de sedação no período pós-operatório foi avaliado por meio de uma escala numérica descritiva (MONTEIRO et al., 2009), conforme descrito a seguir: 0 = nenhuma sedação; 
Tabela 1 - Escala composta de dor da Universidade de Glasgow (modificada por MURREL et al., 2008)

\begin{tabular}{|c|c|c|c|}
\hline \multirow{11}{*}{$\begin{array}{l}\text { Do lado de fora do canil, olhar para o cão e } \\
\text { responder as seguintes questões. }\end{array}$} & Parâmetros & Critério & $\mathbf{P}$ \\
\hline & \multirow{3}{*}{ Postura } & Rígido & 1,20 \\
\hline & & Corcunda & 1,13 \\
\hline & & Normal & 0,00 \\
\hline & \multirow{4}{*}{ Vocalização } & Chora & 0,83 \\
\hline & & Geme & 0,92 \\
\hline & & Grita & 1,75 \\
\hline & & Não vocaliza & 0,00 \\
\hline & \multirow{3}{*}{$\begin{array}{l}\text { Atenção à ferida } \\
\text { cirúrgica }\end{array}$} & Mordendo & 1,40 \\
\hline & & Lambendo/olhando/ esfregando & 0,94 \\
\hline & & Ignora & 0,00 \\
\hline \multirow{5}{*}{$\begin{array}{l}\text { Entre no canil e chame } 0 \text { animal pelo nome. } \\
\text { Encorage- } 0 \text { a se levantar e andar até você. } \\
\text { Avalie a mobilidade. }\end{array}$} & \multirow{5}{*}{ Mobilidade } & Recusa-se a se mover & 1,56 \\
\hline & & Rígido, duro, inflexível & 1,17 \\
\hline & & $\begin{array}{l}\text { Lento ou relutante para levantar e } \\
\text { sentar }\end{array}$ & 0,87 \\
\hline & & Claudicando & 1,46 \\
\hline & & Normal & 0 \\
\hline \multirow{5}{*}{$\begin{array}{l}\text { Avalie, em seguida, a reação do animal à } \\
\text { manipulação da ferida cirúrgica. }\end{array}$} & \multirow{5}{*}{ Resposta ao toque } & Chora & 1,37 \\
\hline & & Foge/tira o corpo fora antes/ao toque & 0,81 \\
\hline & & Tenta morder antes/ao toque & 1,38 \\
\hline & & Rosna/guarda antes/ao toque & 1,12 \\
\hline & & Não reage & 0 \\
\hline \multirow{7}{*}{$\begin{array}{l}\text { Finalmente, dê sua impressão sobre } 0 \\
\text { comportamento e o conforto. }\end{array}$} & \multirow{5}{*}{ Comportamento } & Agressivo/deprimido & 1,22 \\
\hline & & Desinteressado & 1,56 \\
\hline & & Nervoso, ansioso, medroso & 1,13 \\
\hline & & Quieto, indiferente & 0,87 \\
\hline & & Contente/saltitante & 0,08 \\
\hline & \multirow{2}{*}{ Conforto } & Desconfortável & 1,17 \\
\hline & & Confortável & 0,00 \\
\hline
\end{tabular}

Fonte: Dados da pesquisa.

1 = sedação leve, alerta, porém menos ativo; 2 = sedação moderada, sonolento, permanecendo em decúbito, mas conseguindo caminhar; 3 = sedação intensa, muito sonolento e incapaz de caminhar quando encorajado. As avaliações foram realizadas no momento basal (antes da cirurgia) e decorridas 1, 2, 3, 4, 6, 8, 12, 18 e 24 horas após a interrupção da anestesia com isoflurano. Em caso de valores de ECDUG acima de 3,5, realizou-se complementação/resgate analgésico com morfina $(0,5 \mathrm{mg} / \mathrm{kg}, \mathrm{IM})$.

Os dados são apresentados como médias \pm desvios padrão. Para comparações entre os grupos foi utilizada a análise de variância (ANOVA), seguida pela correção de Bonferroni. Para comparações ao longo do tempo, foi realizada a ANOVA e o teste de Dunnet. Foram consideradas diferenças significativas quando $p<0,05$. 


\section{Resultados}

O tempo real decorrido da administração da tranquilização até a indução anestésica foi de $37 \pm 6$ minutos e a dose média de propofol necessária à indução da anestesia foi de 3,9 $\pm 1,1 \mathrm{mg} / \mathrm{kg}$ (médias de todos os grupos). 0 tempo real decorrido da indução anestésica até a administração do tratamento foi de $18 \pm 4$ minutos e o tempo decorrido entre a administração do tratamento até o início da cirurgia foi de $35 \pm 5$ minutos (médias de todos os grupos).

A concentração de isoflurano aumentou em todos os tratamentos durante o procedimento cirúrgico, mas diferenças significativas em relação ao momento basal foram observadas somente no grupo Controle (no momento 3 = M3) e no grupo MX (no M2). Após o aumento inicial, houve redução gradual nessa variável, sendo que no grupo MX, os valores nos M4 e M5 foram significativamente menores do que no basal (Figura 1).

Houve elevação significativa da FC no grupo Controle, mas não nos demais grupos, no momento M2. Todos os grupos apresentaram hipotensão (PAM < $60 \mathrm{mmHg}$ ) no momento Basal. Porém, os valores médios de pressão arterial aumentaram em todos os grupos após o início da cirurgia. Elevação significativa em relação ao basal foi observada em todos os grupos nos valores de PAM e PAD no momento M1. No momento M2, a diferença nos valores de PAM e PAD foi significativa somente no Controle. Houve elevação significativa nos valores de PAS nos grupos Controle e MN no momento M1. Durante todo o procedimento os valores de $\mathrm{ETCO}_{2}$ mantiveram-se entre 30 e $38 \mathrm{mmHg}$ em todos os grupos (Figura 1), valores considerados normais para cães (DIBARTOLA, 2007). No momento basal, o valor médio de temperatura retal de todos os grupos foi de $37,5 \pm 0,6^{\circ} \mathrm{C}$. Houve redução média de, aproximadamente, $1^{\circ} \mathrm{C}$ em todos os grupos ao longo do procedimento cirúrgico.

A duração dos procedimentos (em minutos) nos grupos Controle, M, MN e MX foram: $78 \pm 8,82 \pm 9$, $87 \pm 14$ e $75 \pm 1$ para cirurgia e $133 \pm 5,141 \pm 16$, $150 \pm 18$ e $146 \pm 30$ para anestesia, respectivamente. Não houve diferença entre os grupos nos tempos de cirurgia e anestesia.

Os escores de dor avaliados de acordo com a ECDUG encontram-se na Figura 2. Não houve diferença significativa entre os grupos nos escores de dor. No grupo MN, uma hora após o fim da anestesia, o escore de dor foi mais elevado $(p<0,05)$ do que o basal. Embora não tenham diferido significativamente, os escores de dor nos grupos M, MN e MX foram mais elevados uma hora após a interrupção da anestesia com isoflurano; depois desse período, houve redução gradual. No grupo Controle, os escores de dor mantiveram-se elevados durante as três primeiras horas após a anestesia e diminuíram após quatro horas. Não foi realizado resgate analgésico em nenhum dos animais no presente estudo.

Os maiores valores dos escores de sedação após o término da anestesia foram observados de uma a três horas. Depois desse período, todos os grupos apresentaram redução nos escores durante as 24 horas de observação pós-anestésica. Todos os grupos apresentaram medianas do escore de sedação igual a zero decorridas oito horas do término da anestesia (Figura 2).

\section{Discussão}

Os resultados do presente estudo demonstraram que a administração pré-operatória de morfina e meloxicam nas doses utilizadas proporcionam analgesia pós-operatória adequada em cadelas submetidas à ovariosalpingohisterectomia e à mastectomia radical unilateral, conforme demonstrado pela ausência de resgate analgésico no grupo Controle. Porém, no período intraoperatório, nenhum dos tratamentos foi capaz de abolir a resposta hemodinâmica ao estímulo cirúrgico, caracterizada por elevação na pressão arterial.

A administração da morfina pela via IM proporciona analgesia com duração de três a quatro horas (FANTONI; MASTROCINQUE, 2002). Tendo em vista a duração do procedimento anestésico (inferior a três horas em todos os grupos), é de se esperar que a morfina administrada na MPA ainda apresente efeito analgésico na primeira hora do período pós-operatório. Neste estudo, foram padronizados o tempo decorrido entre a tranquilização e a indução anestésica, o tempo entre a indução e a administração do tratamento experimental e o tempo entre a administração do tratamento e o início da cirurgia. Dessa forma, é improvável que diferenças entre os tratamentos nos escores de dor pós-operatória sejam explicadas por maior ou menor efeito analgésico residual proporcionado pela morfina administrada na tranquilização. 

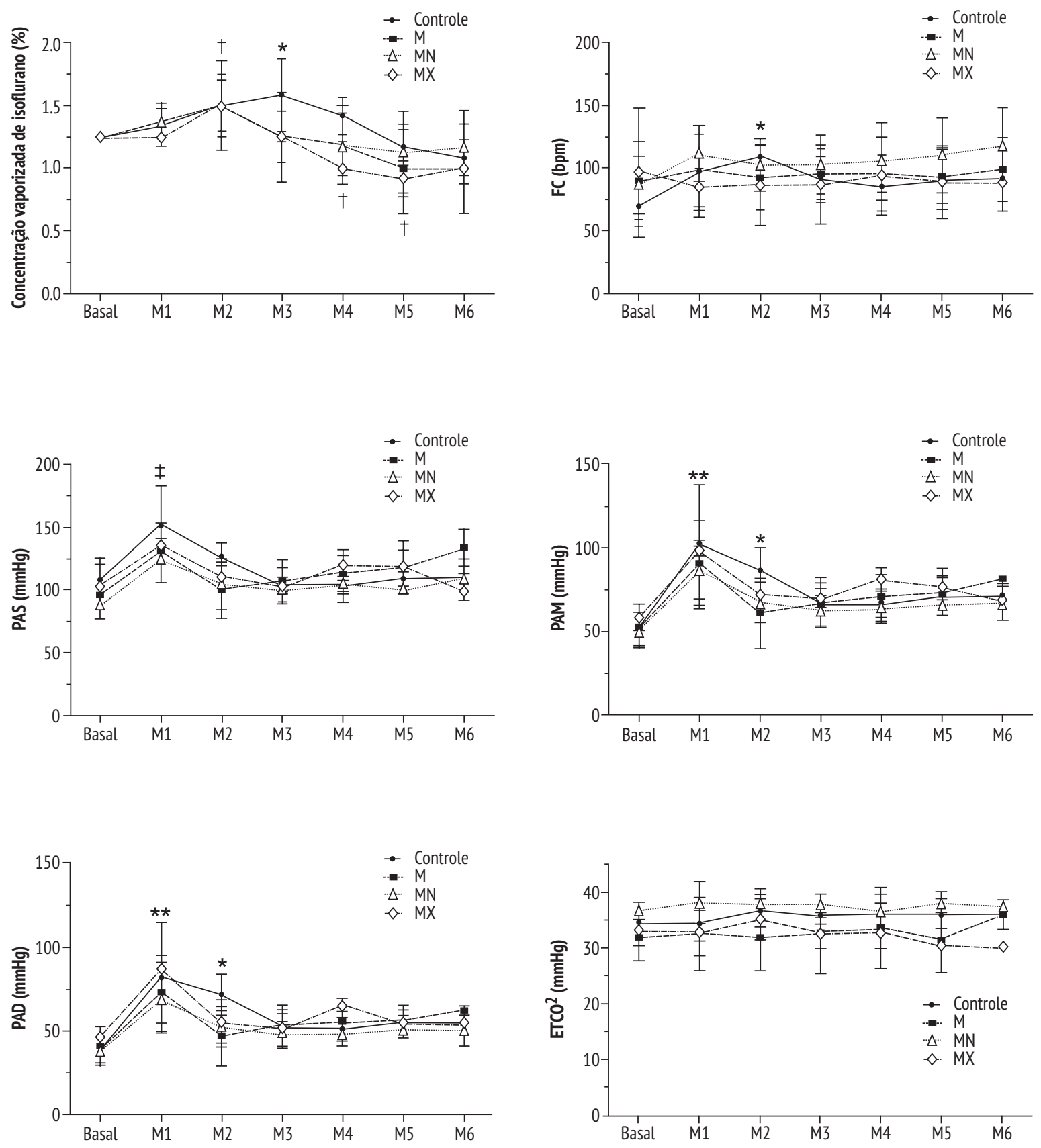

Figura 1 - Valores médios ^ DP das concentrações vaporizadas de isoflurano, frequência cardíaca (FC), pressão arterial sistólica (PAS), pressão arterial média (PAM), pressão arterial diastólica (PAD) e concentração expirada de dióxido de carbono $\left(\mathrm{ETCO}^{2}\right)$ em cadelas após a administração peridural de salina (Controle), morfina (M), morfina + neostigmina (MN) e morfina + xilazina (MX)

Fonte: Dados da pesquisa

Legenda: ${ }^{*}$ = diferença significativa em relação ao basal no Grupo Controle; $\dagger=$ diferença significativa em relação ao basal no Grupo MX; † = diferença significativa em relação ao basal nos Grupos Controle e MN; ** = diferença significativa em relação ao basal em todos os grupos $(p<0,05)$. 

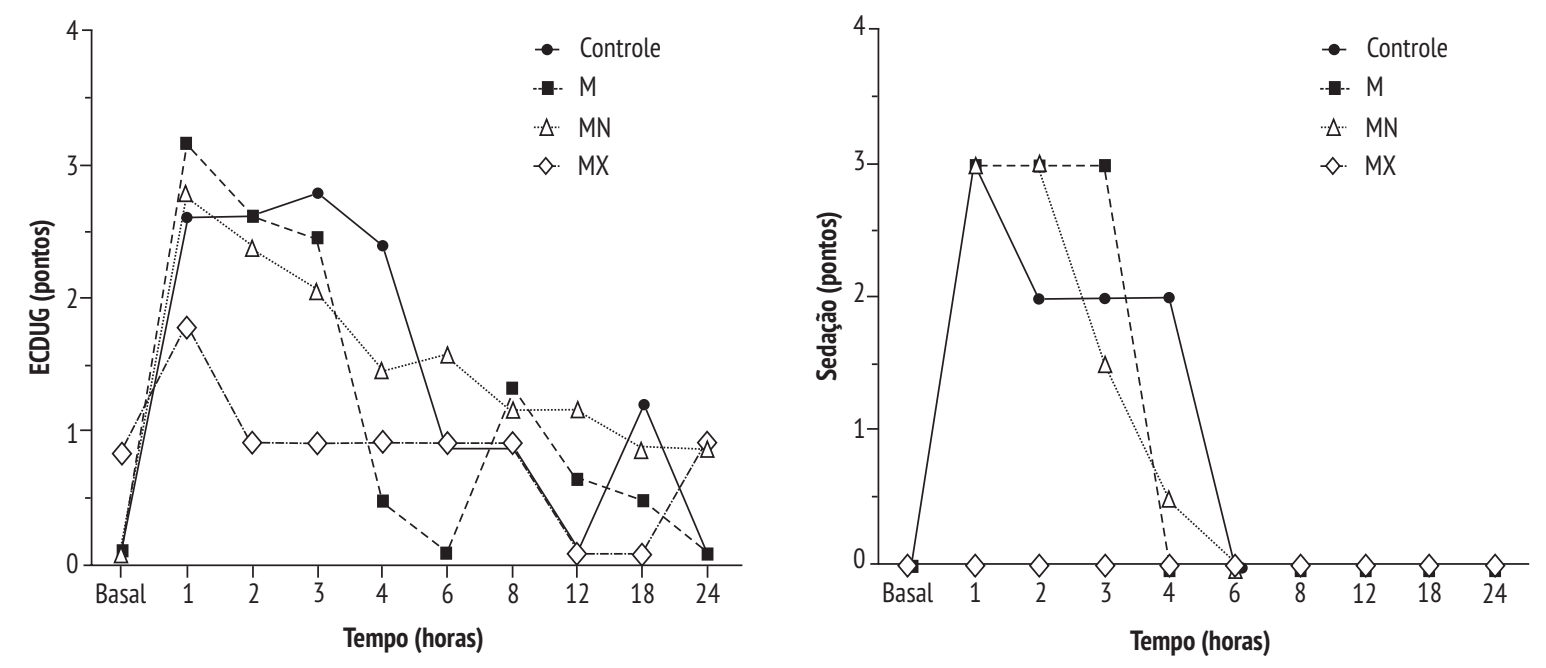

Figura 2 - Medianas dos escores de dor pela Escala Composta de Dor (Universidade de Glasgow - ECDUG) e escores de sedação observados no período pós-operatório em cadelas que receberam a administração peridural de salina (Controle), morfina (M), morfina + neostigmina (MN) e morfina + xilazina (MX)

Fonte: Dados da pesquisa.

Os resultados do presente estudo devem ser avaliados diante de algumas limitações. 0 número de animais em cada grupo $(n=4$ nos grupos $\mathrm{M}$ e MN e $\mathrm{n}=3$ nos grupos Controle e $\mathrm{MX}$ ) pode não ter sido suficiente para demonstrar algumas diferenças reais entre os tratamentos. Entretanto, mesmo com esse número pequeno de animais, foi possível demonstrar diferenças significativas ao longo do tempo em cada grupo. Outra limitação é o fato de as concentrações vaporizadas de isoflurano serem utilizadas ao invés das concentrações expiradas. As concentrações expiradas, mensuradas por um analisador de gases que realiza amostragem contínua da porção distal da sonda endotraqueal, refletem a concentração alveolar dos anestésicos inalatórios. A concentração alveolar se correlaciona com a concentração de anestésico no Sistema Nervoso Central (STEFFEY; MAMA, 2007). Por outro lado, a concentração vaporizada não reflete fielmente a concentração alveolar e uma mesma concentração vaporizada pode resultar em concentrações alveolares diferentes em ocasiões distintas. Para tentar minimizar essa limitação, um mesmo vaporizador calibrado foi usado e o fluxo de oxigênio utilizado foi mantido em 1,5 a 2,0 L/min em todas as ocasiões.

Em todos os grupos experimentais deste estudo, houve hipotensão (PAM $<60 \mathrm{mmHg}$ ) no momento basal. A pressão arterial é o produto da resistência vascular sistêmica (RVS) pelo débito cardíaco (DC), de acordo com a fórmula: PA = RVS $\times$ DC (CUNNINGHAM, 1999). Pode-se sugerir que a hipotensão resultou em virtude do efeito vasodilatador causado pela administração do isoflurano associado à ausência de estímulo cirúrgico no momento basal. Foi relatado anteriormente que, em plano moderado de anestesia, o isoflurano causa diminuição da RVS enquanto o DC é preservado (PAGEL et al., 1991). Portanto, a redução na pressão arterial parece ter sido o resultado da redução na RVS e não no DC. Adicionalmente, a administração da acepromazina pode ter colaborado para a redução da pressão arterial por meio do bloqueio de receptores alfa- 1 adrenérgicos vasculares, levando à diminuição da RVS (MONTEIRO et al., 2007).

Com exceção do tratamento MX, houve elevação da FC em todos os tratamentos após o início do procedimento cirúrgico. A elevação da FC e da pressão arterial, em resposta ao estímulo cirúrgico, resultam do aumento no tônus do sistema nervoso autônomo simpático, com a consequente liberação de catecolaminas na circulação e nos terminais nervosos simpáticos (FANTONI; MASTROCINQUE, 2002). A resposta hemodinâmica ao estímulo nociceptivo 
pode ser abolida por plano anestésico profundo ou pela administração de analgésicos (ILKIW, 1999). Os resultados do presente estudo demonstram que nenhum dos tratamentos estudados foi capaz de abolir a resposta simpática ao estímulo nociceptivo, ocorrendo elevação na PAM em 92, 75, 74 e 66\% nos grupos Controle, M, MN e MX, respectivamente. Esses resultados sugerem que a administração peridural dos tratamentos $\mathrm{M}$, MN e MX foram capazes de atenuar discretamente essa resposta em comparação ao grupo Controle. Adicionalmente, no tratamento MX ocorreu redução na FC em relação ao valor basal. Quando administrados pela via sistêmica, os fármacos da classe dos agonistas alfa-2 adrenérgicos, como a xilazina, causam redução da FC por atuarem em receptores alfa- 2 adrenérgicos centrais, reduzindo o tônus simpático e aumentando o tônus vagal (LEMKE, 2007). Ainda que as doses de agonistas alfa-2 adrenérgicos utilizadas pela via peridural sejam, geralmente, menores do que as utilizadas pela via sistêmica, foram relatados efeitos adversos (como bradicardia e sedação) quando estes fármacos foram administrados pela via espinhal em cães (SABBE et al., 1994; SOARES et al., 2004). Em razão de sua lipossolubilidade, esses fármacos são rapidamente absorvidos para a corrente sanguínea a partir do espaço peridural, causando efeitos sistêmicos (SABBE et al., 1994). Como no presente estudo ocorreu diminuição da FC em relação aos valores basais no grupo MX, estes resultados corroboram com estudos anteriores, nos quais foi relatado que o efeito dos agonistas alfa-2 sobre a FC ocorre mesmo quando esses fármacos são administrados pela via peridural, em virtude da absorção sistêmica da xilazina a partir do espaço peridural (SOARES et al., 2004).

No presente estudo, os valores máximos de pressão arterial foram observados no momento M1, a partir do qual houve redução. Uma vez que o procedimento cirúrgico era sistematicamente iniciado pela ovariosalpingohisterectomia, seguida pela mastectomia radical unilateral, o momento M1 geralmente coincidia com o pinçamento e a ligadura de pedículos ovarianos e uterino, os quais proporcionaram estimulação nociceptiva mais intensa do que a divulsão da cadeia mamária durante a mastectomia.

Gasparini et al. (2007) realizaram um estudo comparando as associações de ropivacaína, lidocaína e xilazina peridural, com a utilização do teste do panículo e do teste térmico a $55^{\circ} \mathrm{C}$ no dorso do animal.
Os autores relataram que a associação da xilazina com um anestésico local promove um bloqueio mais cranial. No presente estudo, a associação da morfina à xilazina não promoveu analgesia suficiente para bloquear o estímulo nociceptivo causado pelo pinçamento dos pedículos ovarianos. Entretanto, a associação da xilazina foi capaz de abolir o aumento da FC e a porcentagem de aumento da PAM no grupo MX foi inferior aos demais (66\% versus $74-92 \%$ nos outros grupos), sugerindo melhor analgesia.

No estudo convencionou-se que escores de dor acima de 3,5 seriam compatíveis com dor moderada, sendo recomendado o resgate analgésico com morfina na dose de $0,5 \mathrm{mg} / \mathrm{kg}$ (MURREL et al., 2008). Um animal do tratamento $\mathrm{M}$ apresentou escore de dor de 5,35 e um animal no grupo MN apresentou escore de 4,5 uma hora após o término da anestesia. Porém, o resgate não foi realizado em nenhuma ocasião. Nas duas ocasiões mencionadas, os animais apresentavam sedação intensa, atribuindo-se pontos nos critérios de mobilidade e comportamento (Tabela 1), resultando em escores de dor artificialmente elevados. Duas horas após a interrupção da anestesia, com os animais apresentando-se menos sedados, os escores de dor foram de 2,43 e 2,33, respectivamente, sugerindo que na primeira hora após a anestesia os escores de dor podem encontrar-se artificialmente elevados em função do grau de sedação residual.

0 fato de que nenhum dos três animais do grupo controle necessitou de resgate analgésico durante 24 horas sugere que a administração preemptiva da morfina $(0,5 \mathrm{mg} / \mathrm{kg})$ associada ao meloxicam $(0,1$ $\mathrm{mg} / \mathrm{kg}$ ) proporcionou analgesia pós-operatória satisfatória em cães submetidos à ovariohisterectomia e mastectomia radical unilateral. Resultado semelhante foi obtido por Nakagawa et al. (2007), ao verificarem que a administração preemptiva de meloxicam $(0,2 \mathrm{mg} / \mathrm{kg}, \mathrm{SC})$ resultou em valores menores de PAS, PAD e PAM em relação ao grupo controle (não tratado com meloxicam).

\section{Conclusão}

A administração preemptiva da morfina, associada ao meloxicam, não inibe a resposta hemodinâmica ao estímulo cirúrgico em cadelas anestesiadas com isoflurano. A administração peridural da morfina, 
isoladamente ou associada à neostigmina ou à xilazina, reduz sensivelmente a resposta hemodinâmica ao estímulo nociceptivo. A administração preemptiva de morfina e meloxicam resulta em analgesia adequada por 24 horas no período pós-operatório de cadelas submetidas à ovariosalpingohisterectomia e mastectomia radical unilateral realizadas durante a anestesia com isoflurano.

\section{Agradecimentos}

À FAPES (Fundação de Amparo à Pesquisa do Espírito Santo), pelo financiamento de uma bolsa de iniciação científica.

\section{Referências}

CARROLL, G. L. Tratamento da dor perioperatória. In: FOSSUM, T. W. et al. (Ed.). Cirurgia de pequenos animais. 2. ed. São Paulo: Roca, 2005. p. 90-99.

CARVALHO, Y. K.; LUNA, S. P. L. Anestesia e analgesia por via epidural em cães - atualização farmacológica para uma técnica tradicional. Clínica Veterinária, ano XII, n. 70, p. 68-76, 2007.

CUNNINGHAM, J. G. As circulações pulmonar e sistêmica. In: CUNNINGHAM, J. G. Tratado de fisiologia veterinária. 2. ed. Rio de Janeiro: Guanabara Koogan, 1999. p. 161-172.

DIBARTOLA, S. P. Introdução aos distúrbios ácidos-básicos. In: DIBARTOLA, S. P. Anormalidades de fluidos, eletrólitos e equilíbrio ácido-básico na clínica de pequenos animais. 3. ed. São Paulo: Roca, 2007. p. 217-238.

FANTONI, D. T.; MASTROCINQUE, S. Fisiopatologia e controle da dor. In: FANTONI, D. T.; CORTOPASSI, S. R. G. (Ed.). Anestesia em cães e gatos. São Paulo: Roca, 2002. p. 323-336.

GASPARINI, S. S. et al. Anestesia epidural com ropivacaína, lidocaína ou associação de lidocaína e xilazina em cães. Efeitos cardiorrespiratório e analgésico. Ciência Rural, v. 37, n. 2, p. 418-424, 2007.

HEDLUND, C. S. Cirurgia dos sistemas reprodutivo e genital. In: FOSSUM, T. W. et al. (Ed.). Cirurgia de pequenos animais. 2. ed. São Paulo: Roca, 2005. p. 610-672.
ILKIW, J. E. Balanced anesthetic techniques in dogs and cats. Clinical Techniques in Small Animal Practice, v. 14, n. 1 , p. $27-37,1999$.

LAURETTI, G. B. et al. Study of three different doses of epidural neostigmine coadministered with lidocaine for postoperative analgesia. Anesthesiology, v. 90, n. 6, p. $1534-1538,1999$.

LEMKE, K. A. Anticholinergics and sedatives. In: TRANQUILLI, W. J. et al. (Ed.). Lumb \& Jones` Veterinary Anesthesia. Iowa: Blackwell Publishing Professional, 2007. p. 203-240.

LOTHE, A. et al. Spinal cholinergic alpha-2 adrenergic interactions in analgesia and hemodynamic control: role of muscarinic receptor subtypes and nitric oxide. The Journal of Pharmacology and Experimental Therapeutics, v. 270, n. 3, p. 1301-1306, 1994.

MARUCIO, R. L. et al. Postoperative analgesic effects of epidural administration of neostigmine alone or in combination with morphine in ovariohysterectomized dogs. American Journal of Veterinary Research, v. 69, n. 7, p. 854-860, 2008.

MONTEIRO, E. R. et al. Effects of acepromazine on the cardiovascular actions of dopamine in anesthetized dogs. Veterinary Anaesthesia and Analgesia, v. 34, n. 5, p. 312-321, 2007.

MONTEIRO, E. R. et al. Comparative study on the sedative effects of morphine, methadone, butorphanol or tramadol, in combination with acepromazine, in dogs. Veterinary Anaesthesia and Analgesia, v. 36, n. 1, p. 25-33, 2009.

MURRELL, J. C. et al. Application of a modified form of the Glasgow pain scale in a veterinary teaching centre in the Netherlands. The Veterinary Record, v. 162, n. 13, p. 403-408, 2008.

NAKAGAWA, K. et al. Influence of preemptive analgesia with meloxicam before resection of the unilateral mammary gland on postoperative cardiovascular parameters in dogs. The Journal of Veterinary Medical Science, v. 69 , n. 9, p. 939-944, 2007.

PAGEL, P. S. et al. Comparison of the systemic and coronary hemodynamic actions of desflurane, isoflurane, halotane and enflurane in the chronically instrumented dog. Anesthesiology, v. 74, p. 539-551, 1991. 
SABBE, M .B. et al. Spinal and systemic action of the $\alpha_{2}$ receptor agonist dexmedetomidine in dogs. Anesthesiology, v. 80 , p. $1057-1072,1994$.

SOARES, J. H. N. et al. Isoflurane sparing action of epidurally administered xylazine hydrochloride in anesthetized dogs. American Journal of Veterinary Research, v. 65 , n. 6, p. 854-859, 2004.

SPINOSA, H. S. Farmacologia do sistema nervoso autônomo. In: FANTONI, D. T.; CORTOPASSI, S. R. G. (Ed.). Anestesia em cães e gatos. São Paulo: Roca, 2002. p. 50-56.

STEFFEY, E. P.; MAMA, K. R. Inhalation anesthetics. In: TRANQUILLI, W. J. et al. (Ed.). Lumb \& Jones' veterinary anesthesia. Iowa: Blackwell Publishing Professional, 2007. p. 355-394.
VALADÃO, C. A. A. et al. Injeção epidural de morfina ou cetamina em cães: avaliação do efeito analgésico pelo emprego de filamentos de von Frey. Arquivo Brasileiro de Medicina Veterinária e Zootecnia, v. 54 , n. 4, p. 383 389, 2002.

Recebido: $26 / 01 / 2011$

Received: 01/26/2011

Aprovado: 23/10/2011

Approved: $10 / 23 / 2011$ 